First publ. in: Journal of the American Chemical Society 132 (2010), 11, pp 3700-3707

Published on Web 02/26/2010

\title{
Polymer-Induced Self-Assembly of Small Organic Molecules into Ultralong Microbelts with Electronic Conductivity
}

\author{
Minghua Huang, ${ }^{\dagger}$ Uwe Schilde,${ }^{\ddagger}$ Michael Kumke,${ }^{\ddagger}$ Markus Antonietti, ${ }^{\dagger}$ and \\ Helmut Cölfen ${ }^{*, \dagger}$ \\ Max-Planck-Institute of Colloids and Interfaces, Colloid Chemistry, Research Campus Golm, \\ D-14424 Potsdam, Germany, and University of Potsdam, Institute of Chemistry, \\ Karl-Liebknecht-Strasse 24-25, D-14476 Potsdam-Golm, Germany
}

Received August 6, 2009; E-mail: coelfen@mpikg.mpg.de

\begin{abstract}
The principle of polymer-controlled crystallization of inorganic materials has been successfully transferred to functional aromatic organic dyes, in this instance 3,4,9,10-perylenetetracarboxylic acid potassium salt (PTCAPS), after its single-crystal structure was determined. The cationic double hydrophilic block copolymer poly(ethylene glycol)-block-branched-poly(ethyleneimine) (PEG-b-PEI) was used as the polymer additive to modify the crystallization of PTCAPS. Ultralong hierarchically structured PTCAPS microbelts with constant width and thickness of each individual belt have been fabricated. The belts are a mesocrystalline assembly of primary nanoparticles with high-energy anionic $\{001\}$ faces stabilized by polymer complexation. Polarization microscopy, X-ray diffraction, optical absorption spectra, and fluorescence spectra indicate the favorable orientation of the 1D microbelts in the close-stacking direction and reveal a specific 1D superstructure fluorescence. Electrical conductivity measurements performed on a single nanobelt disclose in the doped state a remarkably high electronic conductivity and further demonstrate extended, wirelike $\pi-\pi$ interactions along the [020] long axis of the belts. Together with the very large length of the belts and their organic-organic hybrid nanostructure, this makes these organic wires potentially interesting for the field of nano-/micro-optoelectronics.
\end{abstract}

\section{Introduction}

In recent years, research on the self-assembly of onedimensional (1D) nano- and microstructures has attracted considerable attention because promising applications in various fields such as catalysis, energy storage, and optoelectronics are expected. Most of these studies have concentrated on the development of effective methods to prepare 1D structures of a variety of inorganic materials and polymers. ${ }^{1,2}$ Progress in the synthesis of inorganic nanostructures has, however, not been paralleled by a concomitant development of organic structures. In fact, in contrast to their inorganic counterparts, organic systems offer high flexibility in both their physicochemical and self-assembly properties and may cover a complementary range of electrical and optical properties. ${ }^{3}$ Therefore, new routes to the controlled synthesis of crystalline 1D structures of organic molecules remain a challenge. A remarkable exception is the recent fabrication of $1 \mathrm{D}$ structures from large planar aromatic

Max-Planck-Institute of Colloids and Interfaces.

$\doteqdot$ University of Potsdam.

(1) (a) Pan, Z. W.; Dai, Z. R.; Wang, Z. L. Science 2001, 291, 1947. (b) Zhang, J. L.; Du, J. M.; Han, B. X.; Liu, Z. M.; Jiang, T.; Zhang, Z. F. Angew. Chem., Int. Ed. 2006, 45, 1116. (c) Sun, X.; Hagner, M. Chem. Mater. 2008, 20, 2869. (d) Yao, W. T.; Yu, S. H. Int. J. Nanotechnol. 2007, 4, 129.

(2) (a) Jiang, H.; Sun, X.; Huang, M.; Wang, Y.; Li, D.; Dong, S. Langmuir 2006, 22, 3358. (b) Nuraje, N.; Su, K.; Yang, N.; Matsui, H. ACS Nano 2008, 2, 502 .

(3) (a) Zhang, X. J.; Zhang, X. H.; Zou, K.; Lee, C.-S.; Lee, S.-T. J. Am. Chem. Soc. 2007, 129, 3527. (b) Zhao, Y. S.; Fu, H. B.; Hu, F. Q.; Peng, A. D.; Yao, J. N. Adv. Mater. 2007, 19, 3554. molecules. ${ }^{4}$ Nanobelt structures of perylene tetracarboxydiimide have been successfully fabricated through a solvent displacement process. ${ }^{5}$ The formation of a 1D nanofibril structure of arylene ethynylene by a gelation process was also reported. ${ }^{6}$ In these cases, the key step to form a 1D structure is to control and optimize the strong entropic-stacking interactions between the anisometric aromatic disks in combination with the hydrophobic interaction of the side chains. Fine structure control requires balancing two energy contributions, which restricts the approach to aromatic systems with appropriate aliphatic side chains.

Bioinspired morphosynthesis strategies, using organic additives and/or templates with complex functionalization patterns for structure control, are a potential alternative for 1Dnanostructure synthesis and have been explored to form welldefined inorganic materials with unique structures in the nanometer and micrometer range. ${ }^{7}$ Double hydrophilic block copolymers (DHBCs), which consist of a hydrophilic sticking block for the crystal and a second hydrophilic block that

(4) Simpson, C. D.; Watson, M. D.; Müllen, K. J. Mater. Chem. 2004, 14, 494.

(5) (a) Balakrishnan, K.; Datar, A.; Oitker, R.; Chen, H.; Zou, J.; Zang, L. J. Am. Chem. Soc. 2005, 127, 10496. (b) Che, Y.; Datar, A.; Balakrishnan, K.; Zang, L. J. Am. Chem. Soc. 2007, 129, 7234.

(6) Balakrishnan, K.; Datar, A.; Zhang, W.; Yang, X.; Naddo, T.; Huang, J.; Zuo, J.; Yen, M.; Moore, J. S.; Zang, L. J. Am. Chem. Soc. 2006, $128,6576$.

(7) (a) Mann, S.; Ozin, G. A. Nature 1996, 382, 313. (b) Mann, S. Angew. Chem., Int. Ed. 2000, 39, 3392. (c) Xu, A. W.; Ma, Y. R.; Cölfen, H. J. Mater. Chem. 2007, 17, 415. (d) Meldrum, F. C.; Cölfen, H. Chem. Rev. 2008, 108, 4332. 
promotes water dispersion, ${ }^{8}$ have been demonstrated to be effective directing agents for the synthesis of inorganic materials with complex higher order hierarchical structures. ${ }^{9}$ For example, by using DHBCs as structure-directing agents in the mineralization process, highly ordered conelike bundles of $\mathrm{BaCrO}_{4}$ nanofibers with repetitive growth pattern or uniform and very thin $\mathrm{CdWO}_{4}$ nanobelts can be readily generated. ${ }^{10}$ These morphosynthesis strategies, however, have been rarely applied to fabricate the superstructures of organic molecules, and crystallization control of amino acids ${ }^{11}$ and organic pigments ${ }^{12}$ is a remarkable exception.

In this work, we will transfer the bioinspired mineralization principle of polymer-coded nanoparticle organization to induce the self-assembly of a small aromatic molecule into 1D superstructures by adding a specially designed DHBC to control the crystallization. A simple organic dye, i.e. the potassium salt of 3,4,9,10-perylenetetracarboxylic acid (PTCAPS), was selected as a model system. The choice of PTCAPS is based on the following considerations. First, stacking of planar aromatic molecules has been proven to be an effective approach to the formation of 1D superstructures. ${ }^{4}$ More importantly, it also provides a route to tailor the relevant electronic functions. The charge carrier mobility could be enhanced along the fiber direction by optimizing stacking and $\pi-\pi$ interactions. ${ }^{13}$ On the other hand, PTCAPS molecules with carboxylate functional groups are negatively charged in an appropriate $\mathrm{pH}$ range. Therefore, we can control both solubility and interaction with the polymer additive in a subtle fashion just by adjusting the solution $\mathrm{pH}$. It is expected that a simple electrostatic interaction between an oppositely charged polymer additive and the PTCAPS molecule is already sufficient to induce superstructure manipulation, in analogy to the self-assembled structures made by ionic self-assembly (ISA). ${ }^{14}$ In addition, the derivative 3,4,9,10-perylenetetracarboxylic dianhydride (PTCADA) shows intense fluorescence and high photostability and has been widely utilized as a functional molecule in such fields as liquid crystal displays, solar energy conversion, and photoelectron molecular devices. ${ }^{15}$ Crystallizing such functional organic molecules might therefore be significant for optoelectronic applications.

\section{Experimental Section}

All chemicals were of analytical grade and were used as received without further purification. Perylene 3,4,9,10-tetracarboxylic di-

(8) Cölfen, H. Macromol. Rapid Commun. 2001, 22, 219.

(9) (a) Wang, T.; Xu, A.; Cölfen, H. Angew. Chem., Int. Ed. 2006, 45, 4451. (b) Yu, S.; Cölfen, H.; Tauer, K.; Antonietti, M. Nat. Mater. 2005, 4, 51. (c) Xu, A.; Dong, W.; Antonietti, M.; Cölfen, H. Adv. Funct. Mater. 2008, 18, 1307. (d) Peng, Y.; Xu, A.; Deng, B.; Antonietti, M.; Cölfen, H. J. Phys. Chem. B 2006, 110, 2988.

(10) (a) Yu, S. H.; Antonietti, M.; Cölfen, H.; Giersig, M. Angew. Chem., Int. Ed. 2002, 41, 2356. (b) Yu, S. H.; Cölfen, H.; Antonietti, M. Adv. Mater. 2003, 15, 133.

(11) (a) Wohlrab, S.; Cölfen, H.; Antonietti, M. Angew. Chem., Int. Ed. 2005, 44, 4087. (b) Wohlrab, S.; Pinna, N.; Antonietti, M.; Cölfen, H. Chem. Eur. J. 2005, 11, 2903. (c) Ma, Y. R.; Börner, H.; Hartmann, J.; Cölfen, H. Chem. Eur. J. 2006, 12, 7882.

(12) (a) Ma, Y. R.; Mehltretter, G.; Plüg, C.; Rademacher, N.; Schmidt, M. U.; Cölfen, H. Adv. Funct. Mater. 2009, 19, 2095. (b) Zhang, T. K.; Zhu, J. H.; Yao, H. B.; Yu, S. H. Cryst. Growth Des. 2007, 7, 2419.

(13) Che, Y.; Datar, A.; Yang, X.; Naddo, T.; Zhao, J.; Zang, L. J. Am. Chem. Soc. 2007, 129, 6354.

(14) (a) Franke, D.; Vos, M.; Antonietti, M.; Sommerdijk, N. A. J. M.; Faul, C. F. J. Chem. Mater. 2006, 18, 1839. (b) Zakrevskyy, Y.; Faul, C. F. J.; Guan, Y.; Stumpe, J. Adv. Funct. Mater. 2004, 9, 835. (c) Guan, Y.; Zakrevskyy, Y.; Stumpe, J.; Antonietti, M.; Faul, C. F. J. Chem. Commun. 2003, 894.

(15) Wang, Z. S.; Huang, C. H.; Li, F. Y.; Weng, S. F.; Ibrahim, K.; Liu, F. Q. J. Phys. Chem. B 2001, 105, 4230. anhydride (PTCD) was purchased from Aldrich. The tripotassium salt of 3,4,9,10-perylenetetracarboxylic acid (PTCAPS) was synthesized as previously reported. ${ }^{16}$ Poly(ethylene glycol)-blockbranched-poly(ethyleneimine) (PEG-b-PEI-branched; weight average molecular weight $\left(M_{\mathrm{w}}\right)$ of PEG, 5000; $M_{\mathrm{w}}$ of ethyleneimine, 1200) was synthesized as previously reported ${ }^{17}$ via the epoxide after prior removal of low molar mass PEI impurities by dialysis with MWCO $1000 \mathrm{~g} \mathrm{~mol}^{-1}$. All glassware for the crystallization experiments was cleaned and sonicated in ethanol for $5 \mathrm{~min}$, rinsed afterward with distilled water, soaked further with a $\mathrm{H}_{2} \mathrm{O} / \mathrm{HNO}_{3}$ $(65 \%) / \mathrm{H}_{2} \mathrm{O}_{2}(1 / 1 / 1 \mathrm{v} / \mathrm{v} / \mathrm{v})$ solution, then rinsed with distilled water, and finally dried in air with acetone.

Control of the morphologies of PTCAPS assemblies in a mixed solvent was achieved by using the following facile recrystallization method. In a typical procedure, $40 \mathrm{mg}$ of PTCAPS and $4 \mathrm{mg}$ of PEG-b-PEI were added to $4 \mathrm{~mL}$ of mixed solvents $\left(\mathrm{H}_{2} \mathrm{O} /\right.$ ethanol $5 / 7 \mathrm{v} / \mathrm{v}$ ) in a glass bottle. $\mathrm{HCl}$ or $\mathrm{KOH}$ was used to adjust the $\mathrm{pH}$ value of the mixed solution. The PTCAPS solution was then heated to $65{ }^{\circ} \mathrm{C}$ for $10 \mathrm{~min}$ with magnetic stirring. Finally the bottle was cooled naturally to ambient temperature without stirring. After crystallization for 10 days, the crystals were isolated by filtering and carefully washed three times with ethanol and then dried at $40{ }^{\circ} \mathrm{C}$ under vacuum.

Powder X-ray diffraction (XRD) was measured in the reflection mode $(\mathrm{Cu} \mathrm{K} \alpha$ radiation) on a Bruker $\mathrm{D} 8$ diffractometer equipped with a scintillation counter. The SEM measurements were performed on a LEO 1550 GEMINI microscope. Light microscopy and polarized light microscopy images were taken in solution with a Leica DMR B microscope. Transmission electron microscopy (TEM) and selected-area electron diffraction (SAED) was performed on a JEOL 2010 microscope at an acceleration voltage of $200 \mathrm{kV}$. The samples were air-dried on carbon-coated copper grids (PTCAPS belts dispersed into $N$-methyl-2-pyrrolidone (NMP) solution). Atomic force microscopy (AFM) was performed on a NanoScope IIIa device (Veeco Instruments, Santa Barbara, CA) in tapping mode. The samples were spin-coated $(3000 \mathrm{rpm})$ from NMP solution on freshly cleaved mica substrates. UV-vis spectra were recorded using a UVIKON 940/941 dual-beam grating spectrophotometer (Kontron Instruments). In order to eliminate contributions from light scattering, diffuse reflectance spectra of the solid samples were recorded using a Lambda $750 \mathrm{UV} / \mathrm{vis}$ spectrometer (Perkin-Elmer) equipped with an integrating sphere. Fluorescence spectra were obtained for samples dispersed in NMP as well as from the solids themselves. The fluorescence measurements were carried out using a Fluoromax3 spectrofluorometer (Jobin Yvon) or a Spex Fluorolog 1680 spectrophotometer. The fluorescence was excited at $\lambda_{\mathrm{ex}} 430$ and $530 \mathrm{~nm}$, respectively. The spectrofluorometer was operated in the single photon counting mode. All optical measurements were performed at room temperature under ambient conditions. Confocal images were taken with a TCLS confocal laserscanning system attached to an inverse microscope from Leica (Wetzlar, Germany). Electrical conductivity measurements were performed with a Gamry ref 600 potentiostat/galvanostat/ZRA. The two-gold-electrode system was fabricated as previously reported. ${ }^{5 \mathrm{~b}}$ For the measurements under a hydrazine atmosphere, a $5.8 \mathrm{~L}$ desiccator was used. Measurements for the PTCAPS reference crystal after 2, 5, and 10 min revealed comparable data. Control measurements for hydrazine and air showed no conductivity for the electrode system without a bridging crystal. The electrical conductivity can be calculated as $\sigma=1 /[(E / I)(A / L)]$, with $E=$ voltage, $I=$ current, $A=$ cross section of the crystal, and $L=$ distance between the electrodes. Crystals suitable for single-crystal $\mathrm{X}$-ray investigation were grown by slowly cooling a saturated solution of PTCAPS in $65^{\circ} \mathrm{C}$ ethanol/water mixtures. The crystal was embedded in perfluoropolyalkylether oil and immediately mounted on a glass fiber and investigated in the absence of moisture,

(16) Langhals, H. Heteocycles 1995, 40, 477.

(17) Sedlak, M.; Cölfen, H. Macromol. Chem. Phys. 2001, 202, 587. 

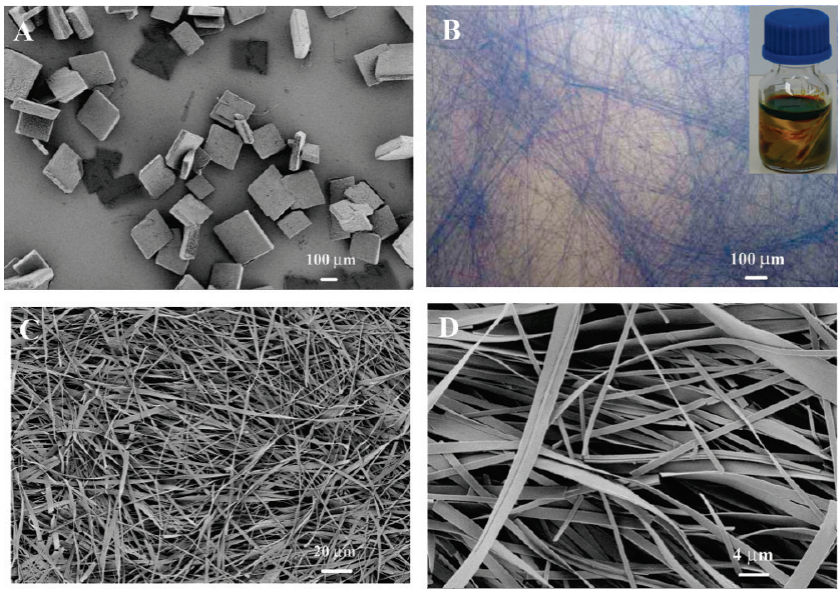

Figure 1. SEM images (A) of PTCAPS crystals without additives and optical microscopy images (B) and SEM images at low magnification (C) and high magnification (D) of PTCAPS crystals in the presence of PEG$b$-PEI $(1.0 \mathrm{~g} / \mathrm{L})$. The inset of (B) gives a photo of PTCAPS belts.

since this leads to partial dissolution of the crystal. X-ray intensity data were recorded at $210 \mathrm{~K}$. The measurements were performed with a IPDS-2 diffractometer (Stoe) with graphite-monochromated molybdenum $\mathrm{K} \alpha$ radiation.

The ORTEP plot and the molecular structure of the perylenetetracarboxylate anion were generated with the program DIAMOND, version 3.1 (Crystal Impact). The unit cell structure was visualized using Mercury, version 2.2, and the modeling of the morphology (Module Morphology) and surface cleavage (Visualizer) were performed with Materials Studio 4.3 software (Accelrys). Wide-angle X-ray scattering (WAXS) patterns were also simulated with the Materials Studio 4.3 software (Accelrys) using the Reflex module.

\section{Results and Discussion}

Ultralong microbelts of PTCAPS with uniform cross-section could be fabricated in the presence of PEG- $b$-PEI as crystal growth modifier. The PEI part of the polymer is cationic under appropriate conditions below its $\mathrm{p} K_{\mathrm{a}}\left(\mathrm{p} K_{\mathrm{a}}\right.$ of PEI $\left.10-11\right),{ }^{18}$ thus making it a good candidate for the controlled interaction with crystal surfaces, while the PEO is water-soluble under the whole range of applied conditions. Due to the fact that the solubility of PTCAPS in water is too high, ethanol/water mixtures were applied (5/7 v/v $\mathrm{H}_{2} \mathrm{O} /$ ethanol) to establish facile recrystallization. PTCAPS dissolves in mixed solvents at $65^{\circ} \mathrm{C}$ and precipitates from the saturated solution at room temperature. In the absence of the polymer additive PEG- $b$-PEI (the default experiment, $\mathrm{pH}$ $8.5)$, the precipitation process of PTCAPS crystals occurred very quickly, and the solution always turned turbid in less than 15 min. After the crystals in contact with the mother liquor were matured for 1 day, tilelike crystals with a size of $100-300 \mu \mathrm{m}$ were obtained (Figure 1A).

This default morphology is not useful for further applications. For a mechanistic understanding of the morphosynthesis experiments, we performed a single-crystal analysis on one of these default crystals. ${ }^{19}$ The coordination environment of the potassium ions and the numbering scheme of the perylenetetracarboxylate anion are given in Figures S1 and S2. Table S1 contains

(18) Gao, Y. X.; Yu, S. H.; Guo, X. H. Langmuir 2006, 22, 6125.

(19) Crystal data for PTCAPS: $M_{\mathrm{r}}=394.46$, triclinic, $P \overline{1}$ (No. 2), $a=$ $7.4228(14) \AA, b=8.1929(18) \AA, c=14.838(4) \AA, \alpha=104.299(18)^{\circ}$, $\beta=100.577(18)^{\circ}, \gamma=95.938(17)^{\circ}, Z=2, D_{\text {calcd }}=1.543 \mathrm{~g} / \mathrm{cm}^{3}$, $T=210(2) \mathrm{K}, R_{1}(I>2 \sigma(I))=0.0359, w R_{2}($ all data $)=0.1034$ $\mathrm{GOF}=1.029, R_{\mathrm{int}}=0.0340$. the crystallographic data and the refinement parameters. Tables S2-S5 give the bond lengths and other structural features. The compound crystallizes in the space group $P \overline{1}$. The asymmetric unit contains only half of the formula unit. Corresponding to this, the other unit is generated by inversion. The inversion center is at the midpoint of the perylene core. The side view in Figure S2 shows that the inequivalent carboxyl C atoms C11 and $\mathrm{C} 12$ deviate by 0.536 and $0.391 \AA$, respectively, from the least-squares plane of the perylene core. The carboxylate groups are torsioned from the perylene ring system-see Figure S2 and Table S4. These deviations of the planes of the carboxylate groups with respect to the mean plane of the perylene core are similar to those in potassium naphthalene-1,4,5,8-tetracarboxylate. ${ }^{20}$ The $\mathrm{C}-\mathrm{O}$ bond lengths of the carboxylate groups range from 1.252(2) to 1.267(2) $\AA$ (Table S2). The two shorter C-O bonds involve the $\mathrm{O}$ atoms closest to the perylene ring system, $\mathrm{O} 1$ and $\mathrm{O} 4$, and the two longer $\mathrm{C}-\mathrm{O}$ bonds involve the $\mathrm{O}$ atoms farthest from the aromatic ring system, O12 and O3. The $\mathrm{O}-\mathrm{C}-\mathrm{O}$ bond angles are widened to $124.8(2)$ and $125.1(2)^{\circ}$, respectively (Table S3). All carboxylate groups are directed outward. This feature can be explained by a comparison of the corresponding $\mathrm{C}-\mathrm{C}-\mathrm{C}$ (carboxylate) angles. The described positions of the carboxylate groups apart and torsioned from the aromatic core are comparable to those in bis(ethylenediammonium) perylene-3,4,9,10-tetracarboxylate octahydrate. ${ }^{21}$

Two unique $\mathrm{K}^{+}$ions have been found. $\mathrm{K} 1$ is coordinated by seven oxygen atoms and $\mathrm{K} 2$ by six oxygen atoms. The range of cation-oxygen distances is 2.653(1)-2.959(2) $\AA$ (Table S2). The coordination geometries are best described as irregular polyhedra. K2 is located in a nearly trigonal-prismatic coordination environment. Two potassium cations share three oxygen atoms. One alternating $\mathrm{O}$ atom arises from a water molecule or from an ethanol molecule. Moreover, the structure of PTCAPS is stabilized by three hydrogen bonds, two between $\mathrm{O} 5 \mathrm{H}$ (water) and $\mathrm{O}$ (carboxylate) and a further one between $\mathrm{O}$ (ethanol) and $\mathrm{O}$ (carboxylate)-for details see Table S5. Furthermore, in the packing a hydrogen-bonded ethanol molecule is incorporated, in which the carbon atoms are disordered over two sites with equal occupancies. The whole structure is shown in Figure S3. Calculation of the equilibrium morphology (Figure S3D) of this crystal under vacuum yields a morphology similar to that observed in Figure 1A, supporting the fact that this morphology is the equilibrium structure.

A completely different morphology is observed after the addition of PEG- $b$-PEI serving as structure-directing agent. The recrystallization of PTCAPS molecules in the presence of 1.0 $\mathrm{g} / \mathrm{L}$ PEG- $b$-PEI at a starting $\mathrm{pH}$ of 8.4 led to shining fibrous structures with a metallic gloss, as shown in the inset of Figure 1B. In fact, the optical microscopy image clearly shows that most of the fibers have a length in the range of millimeters (Figure 1B). Higher magnification SEM images demonstrated that the fibers have a beltlike morphology with a thickness of about $100-200 \mathrm{~nm}$, while the width is about $0.6-2.5 \mu \mathrm{m}$ (Figure 1C,D). However, it is important to emphasize that the width and thickness of each individual nanofiber are constant over the entire fiber length. It should be noted that the produced fibers are easily broken during the preparation of SEM samples. The close-to-rectangular shape of the cross section can also be seen from the broken belts. Polarization microscopy images

(20) Fitzgerald, L. J.; Gallucci, J. C.; Gerkin, R. E. Acta Crystallogr. 1993, C49, 1287.

(21) Li, J.-R.; Tao, Y.; Yu, Q.; Bu, X.-H. Cryst. Growth Des. 2006, 6, 2493. 
(Figure S4) reveal a rather perfect pleiochromism of the fibers, with the transition moment and the long molecular axis of the perylene being oriented perpendicular to the fiber direction. The microbelts can therefore be considered as extended stacks of perylene derivatives, with the maximal conductivity in the fiber direction. This is the technically demanded morphology.

If the $\mathrm{pH}$ was further decreased to 8.1 , no obvious further change for the morphology of the ultralong microbelt structures was found, while increasing the $\mathrm{pH}$ to 9.6 results in more complex, aggregated-sheet-like structures (Figure S5). The effect of other processing parameters such as decreasing crystallization temperature has also been investigated, and only minor changes in the dimensions and the shape of the microbelts were found (see Figure S6). At $\mathrm{pH}$ values of 8.1-8.4, PEG- $b$-PEI possesses a highly positive charge $\left(\mathrm{p} K_{\mathrm{a}}(\mathrm{PEI})=10-11\right)$ and strongly interacts with the highly negative PTCAPS molecules $\left(\mathrm{p} K_{\mathrm{a} 1}(\mathrm{PTCAPS})=5.9, \mathrm{p} K_{\mathrm{a} 2}(\mathrm{PTCAPS})=2.6\right.$ with the latter $\mathrm{p} K_{\mathrm{a}}$ not clearly identified; see Figure $\mathrm{S} 7$ ). The interaction slows down the crystallization process and will expose crystal faces such as $\{001\}$ which are otherwise highly negative, thus of high energy and therefore less exposed in the final morphology according to Wulff's rule. ${ }^{22}$ The conductivity of the supersaturated PTCAPS solution with PEG- $b$-PEI decreased quickly during the first $1 \mathrm{~h}$, followed by a slow decrease during the subsequent precipitation period up to $2.5 \mathrm{~h}$ until the conductivity stays constant, indicating a rapid particle nucleation of secondary, nonmolecular species prior to the slower crystallization (Figure S8). This causes the organized nanoparticle stacking and more extended growth along the belt long axis, with the carboxylate side chain charges pointing to the flat belt faces $\{001\}$ where the polymer is bound, providing also the colloidal stabilization of the belt.

All the above evidence indicates that it is the electrostatic polymer-crystal interaction which drives the formation of the $1 \mathrm{D}$ structure. In the absence of polymer additive, PTCAPS molecules are negatively charged. On one hand, $\pi-\pi$ stacking of perylene aromatic backbones will dominate the molecular packing configuration. On the other hand, the electrostatic repulsion between the negatively charged PTCAPS molecules will prevent the effective packing of PTCAPS molecules along one dimension. At $\mathrm{pH} 8.1-8.4$, the polymer additive PEG- $b$ PEI has a positive charge and strong interaction with the carboxylate side chain charges of PTCAPS. This can screen the electrostatic repulsion between the negatively charged PTCAPS molecules, block further nanoparticle growth at the adsorption site, and allow for more organized molecular stacking and more extended growth of the primary nanoparticles along the belt long axis by $\pi-\pi$ stacking. The role of the electrostatic interaction can be demonstrated by increasing the $\mathrm{pH}$ to 10.3 at fixed polymer concentration. At this $\mathrm{pH}$ value, the interaction between PEG- $b$-PEI and PTCAPS molecules is much weaker since the $\mathrm{pH}$ is very close to the $\mathrm{p} K_{\mathrm{a}}$ of PEI. Indeed, a $1 \mathrm{D}$ structure was no longer formed. Tilelike microcrystals similar to the result of the default experiment were now obtained, indicative of the absence of polymer interaction and crystallization of PTCAPS with the potassium counterion.

Additional insight into the molecular packing and orientation within the belts is obtained from the consideration of the $d$ spacings. XRD patterns of the PTCAPS powder reference sample and the PTCAPS microbelts (Figure 2) show that the microbelts have the same polymorph as that of the PTCAPS

(22) Wulff, G. Z. Kristallogr. 1901, 34, 449.

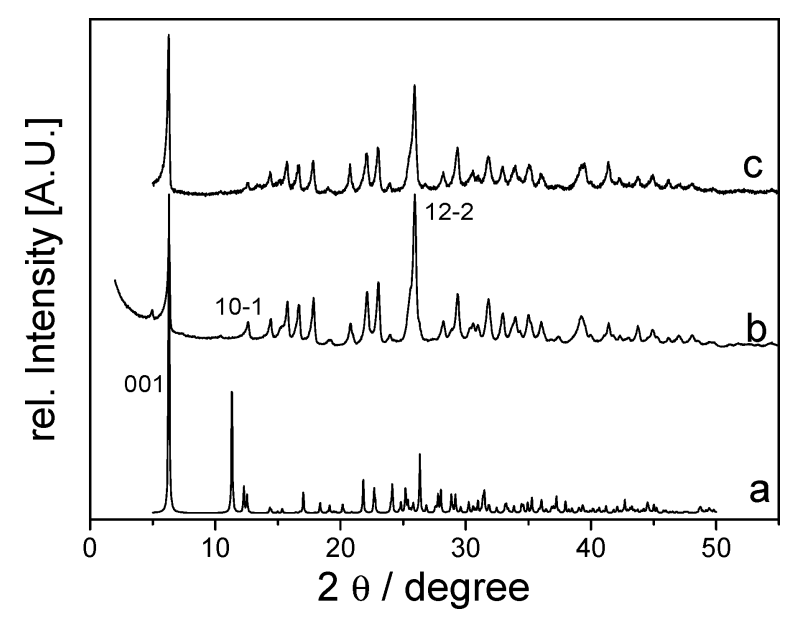

Figure 2. WAXS patterns of PTCAPS calculated curve (a), powder (b), and belts (c). For the sake of clarity, the relative intensity of the calculated WAXS was increased by a factor of 2 and the (001) peak extends through the powder data.

powder. The stacking peak (12-2) of the aromatic units corresponding to a $d$ spacing of $0.34 \mathrm{~nm}$ is clearly seen in both structures. $^{2 \mathrm{a}, 23}$ Also, two peak intensities of the microbelts differ remarkably from that of the powder sample of the default morphology in Figure 1A, namely (001) and (10-1). Surface slices of these faces are shown in Figure S9. The peak for the charged (001) face becomes intensified for the microbelts, indicating increased thickness in [001]. In addition, the peak intensity for [10-1] is less intense in the belts, indicating a decreased thickness compared to the powder reference sample. The $(10-1)$ face is shown as a surface cut in Figure S9 but does not show special features. In fact, the change of peak intensities while keeping the unit cell symmetry clearly underlines the effectivity of the concept of polymer-controlled morphosynthesis as such.

${ }^{1} \mathrm{H}$ NMR characterization of the PTCAPS microbelts dissolved in $\mathrm{D}_{2} \mathrm{O}$ was performed to obtain molecular information on the components of the microbelts (Figure S10A). It can be seen that characteristic PTCAPS signals at ca. $8.5 \mathrm{ppm}^{14 \mathrm{c}}$ are observed in both spectra of the PTCAPS microbelts (Figure S10A) and the PTCAPS powder (Figure S10B), and the signals of PEI (ca. $2.2 \mathrm{ppm})^{24}$ only appeared in the spectrum of the PTCAPS microbelts. This shows that the PTCAPS microbelts are a hybrid of PTCAPS and PEG- $b$-PEI. Due to the fact that the signals of PEI (ca. $2.2 \mathrm{ppm}$ ) and PTCAPS (ca. $8.5 \mathrm{ppm}$ ) are completely separated, it is determined that six PTCAPS molecules are bound to one PEG- $b$-PEI polymer in the belts. Since the applied PEI has on average 28 monomer units with $25 \%$ primary, $50 \%$ secondary, and $25 \%$ tertiary amines, about 7 primary amines would be available for binding of the sterically demanding PTCAPS, with one of the primary amine groups being presumably used for coupling of the PEG block. ${ }^{17}$ This means that practically all of the available six primary amine groups are coordinated to PTCAPS, underlining the strong binding between these two molecules.

To obtain more information about the inner texture of the microbelt hybrid material, an etching experiment was performed

(23) (a) Wei, Z. X.; Laitinen, T.; Smarsly, B.; Ikkala, O.; Faul, C. F. Angew. Chem., Int. Ed. 2005, 44, 751. (b) Ornatska, M.; Peleshanko, S.; Rybak, B.; Holzmueller, J.; Tsukruk, V. V. Adv. Mater. 2004, 16, 2206.

(24) Tian, H. Y.; Deng, C.; Lin, H.; Sun, J. R.; Deng, M. X.; Chen, X. S.; Jing, X. B. Biomaterials 2005, 26, 4209. 

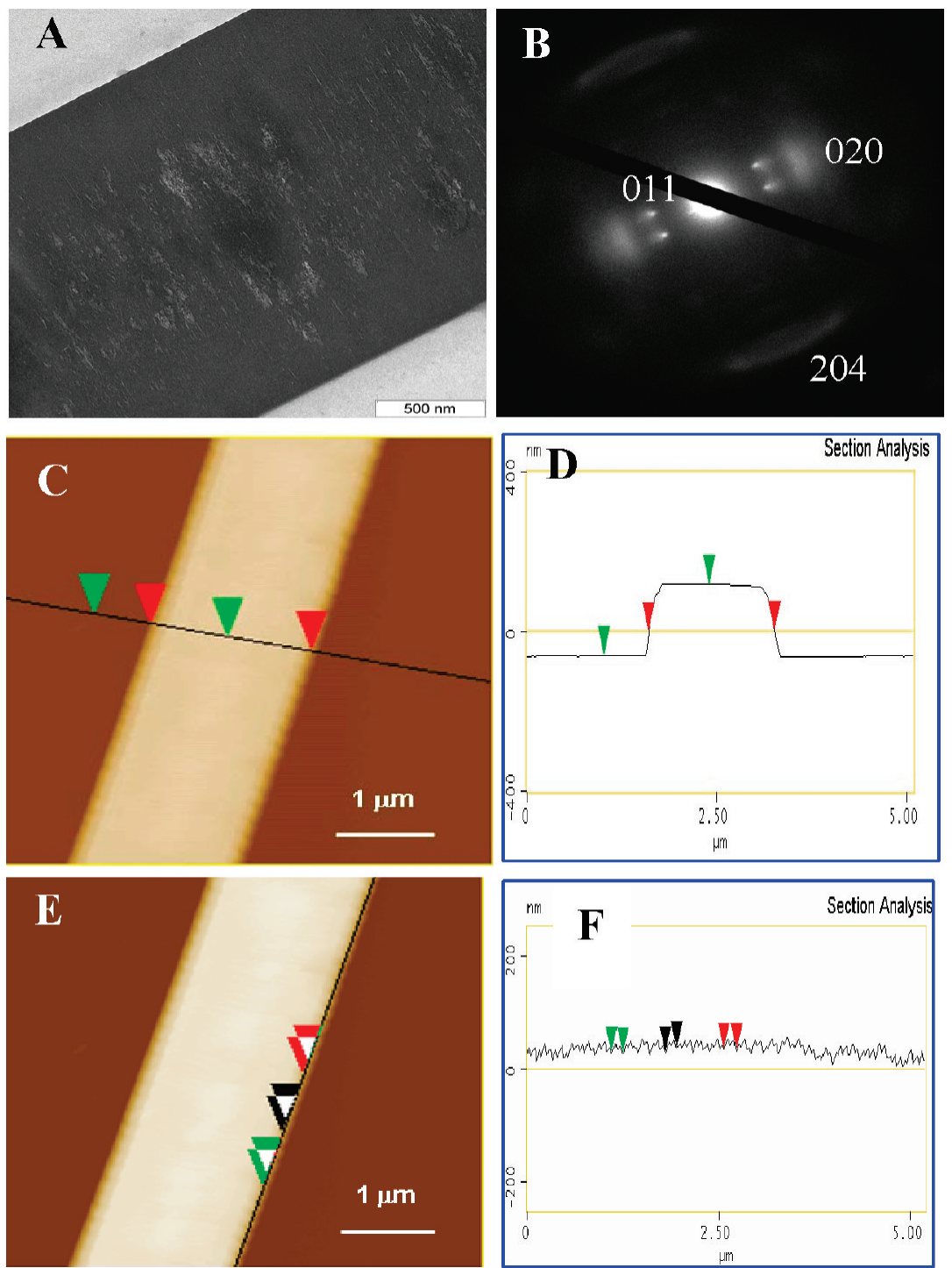

Figure 3. (A) TEM image of a PTCAPS belt cast on carbon film. (B) Selected area electron diffraction of a PTCAPS belt. (C) AFM image of a single belt cast on the mica substrate. (D) Cross section along the black line in (C). (E) AFM image of a single belt as that in (C). (F) Line-scan profile along the black line in $(\mathrm{E})$.

by increasing the $\mathrm{pH}$ value of the belts dispersion to 12 for several minutes. At this $\mathrm{pH}$, which is above the $\mathrm{p} K_{\mathrm{a}}$ of PEI, the block copolymer PEG- $b$-PEI within the crystals should no longer interact with PTCAPS and thus partially dissolve out. The resulting rough structures reveal the inner connectivity of the crystalline superstructure. As shown in Figure S11, SEM results of the etching experiments indeed reveal a porous structure on the side of the belts, indicating the presence of previous PEG$b$-PEI polymer domains.

TEM was used to further examine the morphology of the PTCAPS microbelts. The results are consistent with a uniform beltlike morphology observed by SEM. Figure 3A shows the typical TEM image of a single belt. The selected area electron diffraction (SAED) pattern of the single belt is displayed in Figure 3B. The sharp $(011)(d \approx 0.62 \mathrm{~nm})$ diffraction spots in this SAED image indicate that the belts have a well-oriented joint structure. In the TEM image (Figure 3A), one can see that the ribbon is indeed a mesocrystalline superstructure, is internally heterogeneous, and contains brighter areas or pores. This is a typical feature of an oriented superassembly of primary nanoparticles. ${ }^{25}$ Two weak and diffuse arcs with $d \approx 0.39 \mathrm{~nm}$ (020) in the direction of the microbelt long axis can also be observed. This indicates a disordered [020] direction for the fiber growth with slight misalignments (Figure 4).

The nanoparticulate mesostructure also becomes obvious when analyzing the fracture surface of the broken belts by SEM and TEM (Figure S12). This image of the fracture surface is typical for a sintered polycrystalline film and clearly demonstrates that the obtained PTCAPS crystal is not a conventional single crystal for which a flat cleavage plane should be expected. As shown in Figure 3C,D, AFM measurement of the sample nevertheless revealed a uniform belt structure with a typical width of about $1.5 \mu \mathrm{m}$, a height of about $180 \mathrm{~nm}$, and a flat surface structure (mean roughness $2.54 \mathrm{~nm}$ ), observations which are consistent with those of SEM and TEM measurements. In comparison to a much smoother line-scan profile obtained along the $b$ direction in the middle of the belt (Figure S13), the line-

(25) (a) Cölfen, H.; Antonietti, M. Mesocrystals and Nonclassical Crystallization; Wiley: New York, 2008. (b) Cölfen, H.; Antonietti, M. Angew. Chem., Int. Ed. 2005, 44, 5576. 


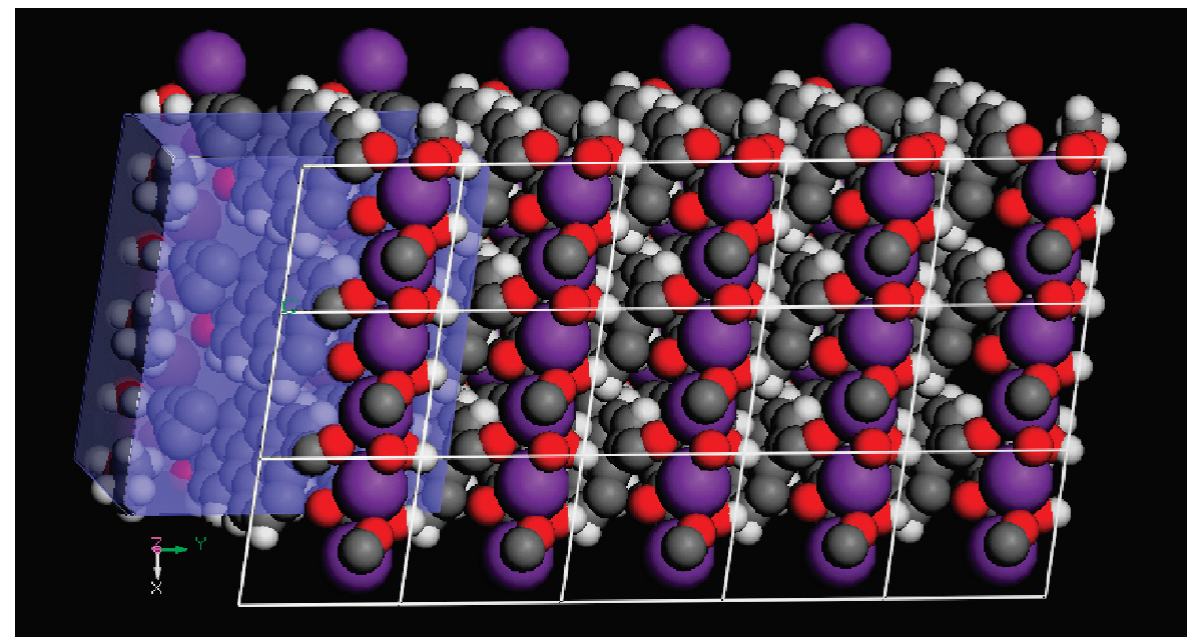

Figure 4. Schematic representation of the microbelt structure extended along the $b$ axis and viewed along the $c$ axis: (gray) carbon; (white) hydrogen; (red) oxygen; (violet) potassium. The default crystal morphology is shown as well as the alignment of the perylene tetracarboxylate potassium salt molecules within the microbelt (not drawn to scale). The white quads are (110) faces. For reasons of clarity, the microbelt structure is drawn with potassium counterions, which are replaced by the block copolymer in the real microbelts.
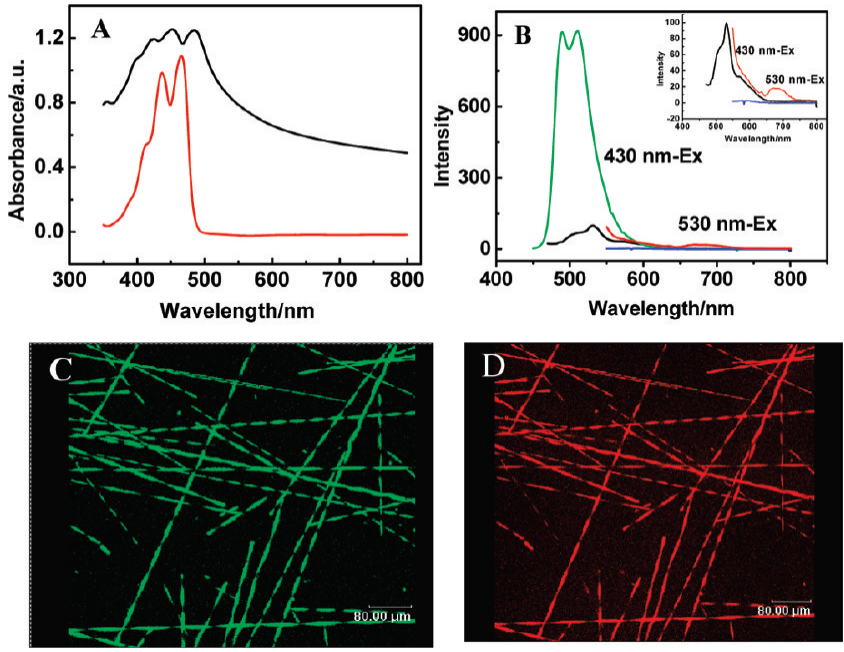

Figure 5. (A) UV-vis spectra of a $45 \mu \mathrm{M}$ PTCAPS solution (red line) and belts dispersed in NMP (black line). (B) Fluorescence emission spectra of $45 \mu \mathrm{M}$ PTCAPS solution excited at $430 \mathrm{~nm}$ (green line) and $530 \mathrm{~nm}$ (blue line), and of a belt suspension excited at $430 \mathrm{~nm}$ (black line) and 530 $\mathrm{nm}$ (red line). Inset: for clarity, the corresponding black, red, and blue lines were extracted. (C) Fluorescence micrograph of the microbelts excited at $488 \mathrm{~nm}$. (D) Fluorescence micrograph of the same microbelts excited at $543 \mathrm{~nm}$.

scan profile along the edge of the belt in Figure 3E clearly reveals a striation pattern which is highly relevant for identification as a mesocrystal (Figure 3F). It is estimated from the AFM line scan at the microbelt edge that the size of a primary nanoparticle building unit is about $50-70 \mathrm{~nm}$, as seen from Figure 3F. From WAXS peaks and using the Scherrer equation, the primary particle size is determined to be $30-40 \mathrm{~nm}$ for the microbelts. This is a reasonable agreement if the dimension of the AFM tip is taken into account.

Packing of functional dyes into a crystal arrangement affects the electronic properties, as most easily demonstrated by UV-vis spectra. Figure 5A shows UV-vis absorption spectra of the PTCAPS molecularly dissolved in water (red line) and of the PTCAPS belts dispersed in $N$-methylpyrrolidone (NMP). The absorption spectrum of the dilute PTCAPS solution shows four relatively narrow absorption peaks at 388, 412, 436, and $465 \mathrm{~nm}$. Upon crystallization, the absorbance peaks of the
PTCAPS belt suspension are red-shifted to 398, 422, 452, and $484 \mathrm{~nm}$, respectively, and the absorption strength at shorter wavelength is relatively enhanced, which should result from the delocalization of the excited state due to $\pi-\pi$ interaction. $^{2 a}$ Also, a line broadening of the peaks is observed for the belt structures, which also reflects the strong intermolecular electronic interactions of the close-packed molecules. ${ }^{26}$ These findings are consistent with data reported for red perylenediimide pigments in the crystalline state. ${ }^{27}$ As shown in Figure S14A, the enhanced $\pi-\pi$ interaction is also seen in the diffuse reflectance spectra of the PTCAPS crystal powder and the belt powder. For the latter an increased absorption in the spectral range of $500 \mathrm{~nm}<\lambda<575 \mathrm{~nm}$ is found, indicating a strong electronic coupling in the belts.

The effects of the 1D nanocrystal superassembly structure on the optical behavior are also seen in the photoluminescence spectra. Figure 5B presents the fluorescence spectra of both a dilute solution of PTCAPS and the belt suspension in NMP at excitation wavelengths of $\lambda_{\mathrm{ex}}=430$ and $530 \mathrm{~nm}$, respectively. At $\lambda_{\mathrm{ex}}=430 \mathrm{~nm}$, in comparison to the spectrum of the dissolved molecules in water (emission peaks at 481 and $509 \mathrm{~nm}$ ), the emission spectrum of the suspended belts seems to be red-shifted to 511 and $531 \mathrm{~nm}$, respectively. These spectral changes may be attributed to $\pi-\pi$ interactions. ${ }^{23}$ In addition, photoluminescence measurements of the suspended belts are prone to inner filter effects (due to high local concentration of perylene chromophores, which is an intrinsic property of the belts), which subsequently also can result in such an alteration of the emission spectrum. Changing the excitation wavelength $\lambda_{\mathrm{ex}}$ from 430 to $530 \mathrm{~nm}$ gives rise to a dual emission for the suspensions of PTCAPS crystals and of the belts in NMP, which further supports the assumption of an increased electronic interaction in the crystalline sample and in the belt structures. ${ }^{23}$ While for a solution of PTCAPS only the regular emission in the green spectral region is observed $\left(\lambda_{\mathrm{ex}}=430 \mathrm{~nm}\right)$ and upon switching the excitation to $\lambda_{\mathrm{ex}}=530 \mathrm{~nm}$ no emission at all is observable, the suspensions of crystalline PTCAPS (figure not shown) as

(26) Luo, Y.; Lin, J.; Duan, H.; Zhang, J.; Lin, C. Chem. Mater. 2005, 17, 2234.

(27) Liu, S. G.; Sui, G.; Cormier, R. A.; Leblanc, R. M.; Gregg, B. A. J. Phys. Chem. B 2002, 106, 1307. 


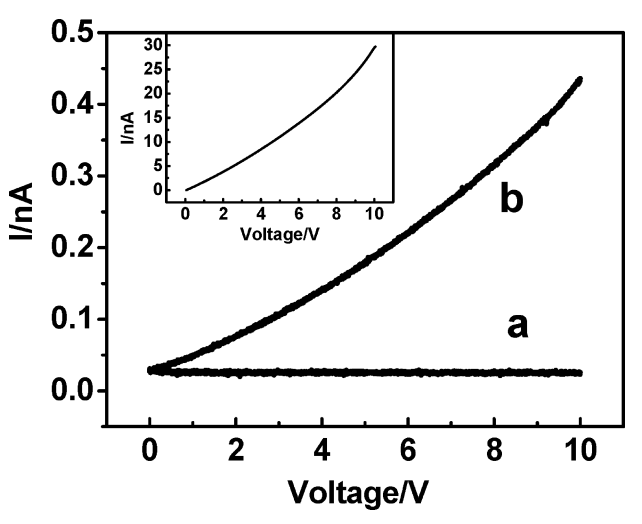

Figure 6. $I-V$ curves measured on PTCAPS belts deposited across a pair of gold electrodes separated by $70 \mu \mathrm{m}$ : (a) in air; (b) under a saturated vapor of hydrazine. The inset shows the $I-V$ curve measured for the reference PTCAPS single crystal under a saturated vapor of hydrazine.

well as of the belts show an additional emission band in the red spectral region (see Figure 5B).

We also performed fluorescence measurements on the PTCAPS crystal powder and the belt powder at $\lambda_{\mathrm{ex}}=530 \mathrm{~nm}$ (Figure S14B). One can see that the red emission is strongest for the PTCAPS crystal powder and much weaker for the belt powder, complementing the results of the diffuse reflectance measurements, in which also for the crystal PTCAPS powder sample a much stronger absorption in the spectral range of 500 $\mathrm{nm}<\lambda<575 \mathrm{~nm}$ was observed. This further underlines the increased electronic interaction present in the belts. Depending on the excitation wavelength used, either the molecular fluorescence typical for the perylene chromophore or the new red fluorescence, which can be attributed to the supramolecular assembly in the solid samples, is excited. The fact that the red emission is distinctly weaker for the belt powder compared to the PTCAPS crystal powder may be interpreted as an increased electronic interaction (e.g., higher number of $\pi$-systems interacting, which is also supported by the results obtained in the following electrochemical investigations, vide infra). Both longer wavelength behavior and lowered efficiency underline the favorable electronic interactions of the close-packed building blocks in the belts and the emergence of a collective property of the organized 1D structure. ${ }^{28}$ The fluorescence microscope images reflect the green and red emissions of identical microbelts more clearly, as shown in Figure 5C,D.

Further support for the extended $\pi-\pi$ interactions along the long axis of the belts can be provided by electrical conductivity measurements. ${ }^{5 \mathrm{~b}}$ Figure 6 shows current-voltage $(I-V)$ measurements of a single microbelt with and without application of hydrazine vapor. Hydrazine, a strong reducing reagent, can donate an electron into PTCAPS through redox reactions. Due to the effective intermolecular $\pi$-electron delocalization along the belts, the doped electrons can migrate rapidly along the belts in the presence of hydrazine. As shown in Figure 6, the current was indeed increased in comparison to the default case without hydrazine, indicating both successful doping and efficient longrange charge migration along the belts. The conductivity obtained from the quasi-linear range at low voltage (up to $5 \mathrm{~V}$ ) was estimated on the basis of the known nanobelt dimensions to be $5.9 \times 10^{-3} \mathrm{~S} \mathrm{~m}^{-1}$ and rises to $10^{-2} \mathrm{~S} \mathrm{~m}^{-1}$ in the range

(28) Datar, A.; Balakrishnan, K.; Yang, X.; Zuo, X.; Huang, J.; Oitker, R.; Yen, M.; Zhao, J.; Tiede, D. M.; Zang, L. J. Phys. Chem. B 2006, $110,12327$. above $8 \mathrm{~V}$, which is comparable to but slightly higher than that reported previously from other planar aromatic organic nanobelts such as perylene tetracarboxydiimide. ${ }^{13}$ Moreover, the value is significantly higher than those reported for other self-assembled molecular nanowires (e.g., acene). ${ }^{29}$ Also, in comparison to other organic semiconductors such as phthalocyanine ${ }^{30 a}$ and nondoped $\mathrm{C}_{60},{ }^{30 \mathrm{~b}}$ the conductivity of the PTCAPS microbelt is greatly increased. In comparison to the conductivity of the reference PTCAPS single crystal (ca. $10^{-6} \mathrm{~S} / \mathrm{m}$, the inset of Figure 6), a 3-4 order of magnitude increase is observed. This large increase in conductivity can in part be related to the higher specific surface of the microbelts due to their mesocrystal structure. Nitrogen adsorption revealed a specific surface area of $18.8 \mathrm{~m}^{2} /$ $\mathrm{g}$, which is about a factor of 2 higher than that of the PTCAPS reference single crystals $\left(8.5 \mathrm{~m}^{2} / \mathrm{g}\right)$. This makes PTCAPS more accessible to electron-donating hydrazine. Coupled with the ultralong length of the belts, their high conductivity reveals some possibilities of the 1D belts in the field of nano-/microoptoelectronics.

\section{Discussion and Conclusion}

In this work, we have successfully transferred the principle of polymer-controlled crystallization in an aqueous environment under ambient conditions from inorganic materials to functional organic dyes and have fabricated hierarchical ultralong microbelts of a perylene dye salt. The favorable formation of the 1D structure is due to the electrostatic complexation with the polymer, which was quantified and allows for exposure of highly charged $\{001\}$ faces and an overall amphiphilic orientation effect of the primary subunits. The microbelt assembly occurs along the $b$ axis, as deduced from $\{020\}$ diffraction spots in SAED in Figure 3B and illustrated in the computer model. The width and thickness directions of the belt are suggested to be [10-1] and [001], which are the two peaks changing their intensities in WAXS displayed in Figure 2, thus indicating a thickness change in these directions as compared to the reference crystal. As mentioned, the addition of the polymer PEG- $b$-PEI can neutralize the electrostatic repulsion between the carboxylate side chain charges of PTCAPS molecules, and the nanoparticles can stack along [001] with intermediate polymer layers. This is visualized in Figure 7 (see also Figure 3B and corresponding discussion). Thus, the peak intensity of the microbelts for the charged (001) face is enhanced.

Since the [020] direction is the one for $\pi-\pi$ stacking and this face has a low affinity for the charged polymer additive, the nanoparticles will likely fuse together in this direction by the process of oriented attachment. ${ }^{31}$ The corresponding nanoparticle arrangement together with the primary particle morphology and face indices is shown in Figure 7. This nanoparticle arrangement is supported by the high conductivity along [020], which requires a continuous $\pi-\pi$ system. This oriented assembly direction is, as far as primary crystal symmetry allows, the direction of maximal polarization interaction between the primary nanoparticles, as already observed for other dye nanorods. ${ }^{32}$ This allows for extended $\pi-\pi$ interactions along

(29) Wakayama, Y.; Hayakawa, R.; Chikyow, T.; Machida, S.; Nakayama, T.; Egger, S.; Oteyza, D. G.; Dosch, H.; Kobayashi, K. Nano Lett. 2008, 8, 3273 .

(30) (a) El-Nahass, M. H.; Farid, A. M.; Attia, A. A.; Ali, H. A. M. Appl. Surf. Sci. 2006, 252, 7553. (b) Popok, V. N.; Jönsson, M.; Campbell, E.E. B. J. Nanosci. Nanotechnol. 2007, 7, 1434.

(31) Penn, R. L.; Banfield, J. F. Geochim. Cosmochim. Acta 1999, 63, 1549.

(32) Taden, A.; Landfester, K.; Antonietti, M. Langmuir 2004, 20, 957. 

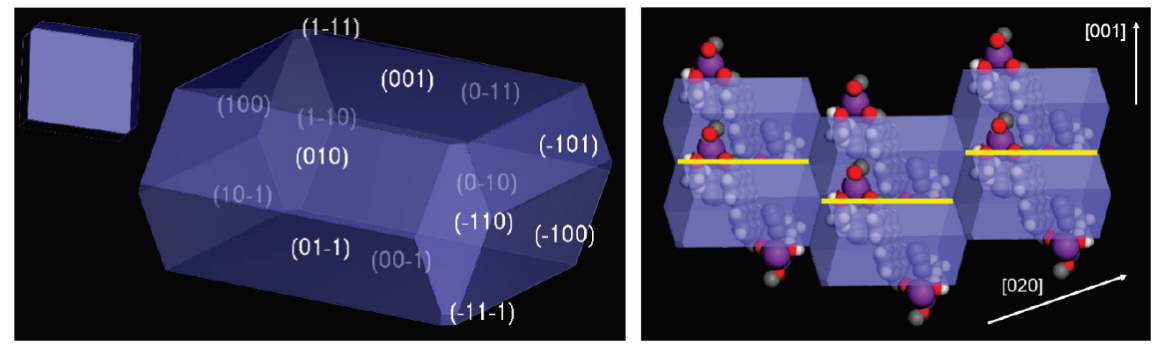

Figure 7. (left) Equilibrium morphology of PTCAPS calculated under vacuum by MS-Modeling (Accelyrys). The inset is the morphology viewed along [001]. (right) Mesocrystal formation by nanoparticle attachment along [020]. One PTCAPS molecule is drawn per nanocrystal (not to scale) to show the orientation of the molecules. The yellow line indicates the block copolymer layers between the nanocrystals, which replace the potassium ions.

the primary microbelt axis due to an optimal positioning of the aromatic ring system.

Possibly relevant for the present observations is that PTCAPS is not fully neutralized by the treatment of the dianhydride with $\mathrm{KOH}$. The titration curve in Figure S7 shows one clearly identifiable leftover $\mathrm{p} K_{\mathrm{a}}$ value, and determination of the potassium content by ICP-MS yielded a potassium content of slightly higher than three potassium/perylene tetraacetic acid units. Regardless if one or two carboxyl groups remain non-neutralized, this affects the dye structure with an orienting electric dipole, as shown for the tripotassium salt in Figure S15. As the corresponding dipole moment in case of a ferroelectric alignment in the nanocrystal will increase linearly with the dimensions of the crystal, a self-limiting growth of the mesocrystal becomes understandable, in a manner very similar to the self-limiting growth observed for $\mathrm{BaCrO}_{4}$ fibers. ${ }^{33}$ The same considerations are true for a mesocrystal. Due to the orientation of the perylene molecules in the crystal, the dipole moment is more effective in the $a$ and $c$ directions (Figures 7 and S15) rather than the microbelt $b$ axis growth direction, which is primarily determined by the $\pi-\pi$ interactions and/or polarization forces. This makes it understandable why the width and thickness of the microbelts, although secondary superstructures, is so defined in the $a$ and $c$ directions within the same belt. Due to the slightly tilted orientation of the perylene units in the microbelt structure and the coupled angle of the dipole moment with the main axis of the belt, the microbelt mesocrystals are not expected to be stable on the long term but they have to rearrange to minimize the dipole-dipole interactions. This is indeed observed with time as a beginning well-defined spiraling of the microbelts, as already depicted in Figure 5.

The presented ultralong crystalline 1D structures exhibit emerging electronic features different from those of the ordinary crystals, as illustrated by optical absorption and fluorescence properties. In addition, we have shown that charge carrier mobility was remarkably improved. ${ }^{34}$ In the presence of electron donors such as hydrazine, the extended $\pi$-electron delocalization along the belts enables significant electrical conductivity along the long axis within each single belt, which is about $3-4$ orders of magnitude higher than that of the single crystal. This could partially be explained by the higher microbelt porosity and thus better accessibility to the electron-injecting hydrazine. Due to the possibility that such microbelts can be conveniently handled,

(33) Yu, S. H.; Cölfen, H.; Antonietti, M. Chem. Eur. J. 2002, 8, 2937.

(34) Grismadale, A. C.; Müllen, K. Angew. Chem., Int. Ed. 2005, 44, 5592. oriented, and spun, the resulting systems may find interesting applications in new types of optoelectronic microdevices.

From a synthetic point of view, we expect to be able to extend the biomimetic mineralization approach to the design and fabrication of 1D structures of other charged, aromatic molecules. With careful choice of a polymer to address different polymer-crystal interactions (such as hydrogen bonding and $\pi-\pi$ stacking), the approach should be also suitable to design other interesting crystalline assemblies from species other than charged organic molecules.

In summary, we have introduced a very simple and upscalable synthesis method for the production of very long microbelts with constant width and thickness in each microbelt, which show significant electrical conductivity along the belt axis as well as fluorescence. For the first time, organic modifications of the organic ring system are no longer necessary to induce its crystallization into a 1D structure. In addition, the composite structure of the microbelts offers unique new possibilities such as targeted doping of the organic semiconductor microbelts and thus significant alteration of their electronic properties and great influence on potential applications. Since the main driving force for the directed self-organization is electrostatic, our approach should be versatile and transferable to other polymer additives with appropriate polyelectrolyte moieties with the associated opportunities to tune the materials properties of the composite material.

Acknowledgment. We acknowledge financial support by the Max Planck Society and thank Shuo Bai for confocal fluorescence microscopy measurements. Anne Heilig is acknowledged for the AFM measurements. M. H. Huang thanks the Alexander von Humboldt Foundation for granting a research fellowship.

Note Added after ASAP Publication. A correction was made in the description of the recrystallization method in the version published March 17, 2010.

Supporting Information Available: Figures, tables, text, the titration curve of PTCAPS, microscopy measurements, conductivity data, ${ }^{1} \mathrm{H}$ NMR data, the unit cell of PTCAPS and a CIF file, the modeling of the default PTCAPS morphology, the modeling of the PTCAPS belts, and X-ray structural data. This material is available free of charge via the Internet at http:// pubs.acs.org.

JA906667X 